\title{
Artigo/Article
}

\section{Evidência de transmissão de leishmaniose visceral por Lutzomyia cruzi no município de Jaciara, Estado de Mato Grosso, Brasil}

\author{
Evidence of transmission of visceral leishmaniasis by Lutzomyia cruzi in the municipality of \\ Jaciara, State of Mato Grosso, Brazil
}

\section{Nanci Akemi Missawa ${ }^{1}$, Márcia Aurélia Esser Veloso ${ }^{1}$, Giovana Belem Moreira Lima Maciel ${ }^{1}$, Érika Monteiro Michalsky ${ }^{2}$ e Edelberto Santos Dias ${ }^{2}$}

\section{RESUMO}

Introdução: O município de Jaciara foi classificado em 2003, como área de transmissão de leishmaniose visceral em situação de surto. O trabalho objetivou determinar evidência de transmissão de Leishmania (Leishmania) infantum chagasi por Lutzomyia cruzi no município de Jaciara, Estado de Mato Grosso, Brasil. Métodos: O município situa-se a $127 \mathrm{~km}$ da capital Cuiabá e é um importante ponto de atração para os praticantes de eco-turismo. Fêmeas de Lutzomyia cruzi, capturadas com armadilha de $\mathrm{CDC}$, foram dissecadas para confirmação da espécie e armazenadas a $-20^{\circ} \mathrm{C}$ em pools de 10 indivíduos para extração de DNA, PCR genérico, RFLP específico e eletroforese em gel de poliacrilamida. Resultados: O levantamento entomológico demonstrou a ocorrência abundante de Lutzomyia cruzi e ausência de Lutzomyia longipalpis, principal vetora da Leishmania (Leishmania) infantum chagasi. Uma das três amostras analisadas apresentou banda característica de DNA de Leishmania (120pb) em PCR genérico. Para confirmação da espécie de Leishmania, na RFLP utilizaram-se controles positivos de Leishmania (Leishmania) amazonensis, Leishmania (Viannia) braziliensis e Leishmania (Leishmania) infantum chagasi digeridas com enzima de restrição HaeIII. Constatou-se um padrão de bandas semelhante à Leishmania (Leishmania) infantum chagasi em uma amostra, confirmando a detecção de infecção natural de Leishmania (Leishmania) infantum chagasi em Lutzomyia cruzi. Conclusões: A ocorrência de casos humanos e cães positivos, a presença da Lutzomyia cruzi e a ausência de Lutzomyia longipalpis, bem como a detecção de infecção natural por Leishmania (Leishmania) infantum chagasi, evidenciam a participação de Lutzomyia cruzi na transmissão da leishmaniose visceral em Jaciara, Estado de Mato Grosso, Brasil.

Palavras-chaves: Lutzomyia cruzi. Leishmania (Leishmania) infantum chagasi. Infecção natural. Mato Grosso

\begin{abstract}
Introduction: The municipality of Jaciara was classified in 2003 as a transmission area for visceral leishmaniasis in outbreak situations. This study aimed to establish evidence of transmission of Leishmania (Leishmania) infantum chagasi by Lutzomyia cruzi in the municipality of Jaciara, State of Mato Grosso, Brazil. Methods: This municipality is located $127 \mathrm{~km}$ from the state capital (Cuiabá) and is an important center for ecotourism practitioners. Females of Lutzomyia cruzi, captured using $\mathrm{CDC}$ traps, were dissected to confirm the species and stored at $-20^{\circ} \mathrm{C}$ in pools of 10 individuals for DNA extraction, generic PCR, specific RFLP and electrophoresis on polyacrylamide gel Results: The entomological survey showed abundant occurrence of Lutzomyia cruzi and absence of Lutzomyia longipalpis (the main vector for Leishmania (Leishmania) infantum chagasi). One of the three samples showed a characteristic DNA band from Leishmania (120 bp) in generic PCR. To confirm the Leishmania species via RFLP, positive controls for Leishmania (Leishmania) amazonensis, Leishmania (Viannia) braziliensis and Leishmania (Leishmania) infantum chagasi digested with restriction enzyme HaeIII were used. A pattern of bands similar to Leishmania (Leishmania) infantum chagasi was found in one sample, thus confirming the detection of natural infection with Leishmania (Leishmania) infantum chagasi in Lutzomyia cruzi. Conclusions: The occurrences of positive cases in humans and dogs, the presence of Lutzomyia cruzi and the absence of Lutzomyia longipalpis together with the detection of natural infection with Leishmania (Leishmania) infantum chagasi, indicate that Lutzomyia cruzi participates in visceral leishmaniasis transmission in Jaciara, State of Mato Grosso, Brazil.
\end{abstract}

Keywords: Lutzomyia cruzi. Leishmania (Leishmania) infantum chagasi. Natural infection. Mato Grosso.

1. Laboratório de Entomologia, Secretaria de Estado de Saúde de Mato Grosso, Cuiabá, MT. 2. Laboratório de Leishmanioses, Centro de Pesquisas René Rachou, Fundação Oswaldo Cruz, Belo Horizonte, MG.

Endereço para correspondência: Dra. Nanci Akemi Missawa. Lab. Entomol/SES/MT. Av. Adauto Botelho s/no, Parque da Saúde, Bairro Coophema, 78085-200 Cuiabá, MT.

Tel: 5565 3661-2934

e-mail: nanci.am@terra.com.br

Recebido para publicação em 28/06/2010

Aceito em 06/10/2010

\section{INTRODUÇÃO}

Aleishmaniose visceral (LV) que era inicialmente uma doença rural passou a acometer áreas urbanas de pequeno e médio porte e, atualmente, ocorre em grandes centros urbanos, revelando o processo de periurbanização e urbanização da doença.

No ano de 2003, o município de Jaciara/MT foi definido como área de transmissão de LV em situação de surto, segundo o Manual de Vigilância e Controle da $L V^{1}$, pois apresentou transmissão, com número de casos superior ao esperado.

Tendo em vista a situação apresentada no município, a Secretaria Estadual de Saúde de Mato Grosso (SES/MT) realizou um levantamento entomológico para verificar a presença do vetor no local, e as pesquisas corroboraram com resultados de Missawa e Lima ${ }^{2}$ que detectaram apenas Lutzomyia cruzi como potencial vetora da LV no município, indicando ser o cerrado, o ambiente preferencial da espécie. Foi registrada a ocorrência de $L$. cruzi e a ausência de $L$. longipalpis, considerado como principal vetor do agente da LV, no Brasil ${ }^{3}$.

A espécie L. cruzi foi incriminada como vetora da Leishmania (Leishmania) infantum chagasi em Mato Grosso do Sul, pois na região de Corumbá e Ladário não ocorria L. longipalpis no período estudado, aliado ao fato de que o flebotomíneo foi encontrado com infecção natural por L. (L.) infantum chagasi ${ }^{4,5}$. Novos conhecimentos sobre a ecologia de $L$. cruzi e sua eventual infecção natural por L. (L.) infantum chagasi em outras regiões trariam informações sobre a importância da espécie como transmissora de LV.

\section{MÉTODOS}

O município de Jaciara/MT situa-se a $127 \mathrm{~km}$ da capital Cuiabá, possui 23.804 habitantes ${ }^{6}$, extensão territorial de $1.801,41 \mathrm{~km}^{2}$, apresenta solo podzólico vermelho amarelo e clima tropical quente e semi-úmido, com quatro meses de seca, de maio a agosto. A precipitação anual é de $1.750 \mathrm{~mm}$, com intensidade máxima em dezembro, janeiro e fevereiro. 
A temperatura média anual é de $22^{\circ} \mathrm{C}$, sendo maior máxima de $40^{\circ} \mathrm{C}$, e a menor $0^{\circ} \mathrm{C}^{6}$. Além do desenvolvimento econômico ligado à agricultura, o município propicia atividades de eco-turismo e lazer.

Foram utilizados dados entomológicos secundários disponibilizados pelo Laboratório de Entomologia da SES/MT, obtidos de levantamentos pontuais realizados em novembro de 2003, janeiro, agosto e setembro de 2004 e do monitoramento de flebotomíneos realizado no período de abril de 2005 a junho de 2006. As coletas de flebotomíneos foram realizadas no peridomicílio de residências com notificação de caso humano e/ou canino de LV. Foram utilizadas armadilhas de luz do tipo CDC instaladas no entardecer e recolhidas cerca de $12 \mathrm{~h}$ após, durante o amanhecer. Os espécimes foram triados, clarificados e identificados segundo a chave de identificação de Young e Duncan ${ }^{10}$, e estão depositados no Laboratório de Entomologia da SES/MT.

Fêmeas de L. cruzi foram dissecadas para confirmação da espécie e armazenadas à $-20^{\circ} \mathrm{C}$ em três pools de 10 indivíduos para posterior análise molecular para detecção de DNA de Leishmania spp. O DNA foi extraído por maceração em microtubo, utilizandose pistilo de plástico, seguido pela adição de $35 \mu l$ de tampão de lise (100mM TRIS-HCl, $100 \mathrm{mM} \mathrm{NaCl}, 25 \mathrm{mM}$ EDTA, 0,5\% SDS, $\mathrm{pH} 8,0)$ seguido de outra maceração. As amostras foram digeridas durante a noite a $37^{\circ} \mathrm{C}$ com proteinase $\mathrm{K}(1,25 \mu \mathrm{l}$ de solução $10 \mathrm{mg} / \mathrm{ml}$ ) e o DNA foi extraído com fenol-clorofórmio ${ }^{7}$. O pellet de DNA foi ressuspendido em $20 \mu \mathrm{l}$ de TE $(10 \mathrm{mM}$ TRIS-HCl pH 8.0, 1mM EDTA). Utilizou-se $10 \mu \mathrm{l}$ para estimar a pureza e a concentração em espectrofotômetro a 260 e $280 \mathrm{~nm}$. As amostras de DNA foram acondicionadas em freezer a $-20^{\circ} \mathrm{C}$, até o momento do uso.

O DNA extraído foi amplificado por procedimento hot start PCR em um termociclador. A mistura de reação foi preparada com $10 \mu l$ de tampão de PCR (100mM TRIS- $\mathrm{HCl}, 500 \mathrm{mM} \mathrm{KCl}$, $15 \mathrm{mM} \mathrm{MgCl}, \mathrm{pH} 9,0), 5 \mu \mathrm{l}$ dNTPs (2mM cada), $2 \mu \mathrm{l}$ de cada iniciador $(200 \mathrm{ng} / \mu \mathrm{l}), 0,5 \mu \mathrm{l}$ de Taq DNA polimerase $(2,5 \mathrm{U} / \mu \mathrm{l})$ e $26,5 \mu l$ de água ultra pura. Adicionou-se $2 \mu \mathrm{l}$ de DNA $(10 \mathrm{ng} / \mu \mathrm{l})$ por reação e a amplificação do DNA foi realizada para Leishmania sp, utilizando-se os iniciadores ${ }^{7}$ : 5' GGG GAG GGG CGT TCT GCG AA 3'; 5'CCG CCC CTA TTT TAC ACC AAC CCC 3'; 5'GGC CCA CTA TAT TAC ACC AAC CCC 3'. Os ciclos de amplificação consistiram em um passo de desnaturação inicial a $94^{\circ} \mathrm{C}$ por $5 \mathrm{~min}$, seguida por 35 ciclos de desnaturação a $94^{\circ} \mathrm{C}$ por $30 \mathrm{~s}$, anelamento a $50^{\circ} \mathrm{C}$ por $30 \mathrm{~s}$; extensão a $72^{\circ} \mathrm{C}$ por 30 s e uma extensão final a $72^{\circ} \mathrm{C}$ por $10 \mathrm{~min}^{8}$. Controles negativos e positivos foram utilizados em todos os experimentos. Para a detecção do DNA da Leishmania sp foi utilizado o método de eletroforese em gel de poliacrilamida (SDS-Page) ${ }^{9}$.

As amostras positivas no PCR genérico foram submetidas à reação de polimorfismo de comprimento dos fragmentos de restrição (RFLP). As amostras referência de Leishmania utilizadas foram Leishmania (Leishmania) amazonensis (IPLA/BR/67/ $\mathrm{PH} 8$ ), Leishmania (Viannia) braziliensis (MHOM/BR/75/ M2930) e Leishmania (Leishmania) infantum chagasi (MHOM/ $\mathrm{BR} / 74 / \mathrm{PP} / 75)$. Os fragmentos de restrição foram separados em gel de poliacrilamida na concentração de $10 \%$.

\section{RESULTADOS}

Foram identificados 3.110 espécimes, pertencentes aos gêneros Lutzomyia e Brumptomyia: Lutzomyia acantopharynx, L. antunesi, L. carmelinoi, L. carrerai carrerai, L. cruzi, L. dasymera, L. davisi, L. evandroi, L. hermanlenti, L. lenti, L. longipennis, L. punctigeniculata, L. sallesi, L. sordellii, L. teratodes, L. termitophila e L. whitmani e B. brumpti.

L. whitmani (66,5\%) e L.cruzi (28,6\%) foram as mais abundantes coletadas no município, com predominância de machos em ambas as espécies. As espécies foram capturadas em todos os meses pesquisados. Não foram capturados espécimes de L. longipalpis no município.

A detecção da Leishmania sp em L. cruzi foi realizada utilizandose marcador de 100 pares de base (Ladder) e controle positivo. Observou-se que a amostra 3 apresentou banda característica de DNA de Leishmania (120pb) conforme demonstrado na Figura 1.

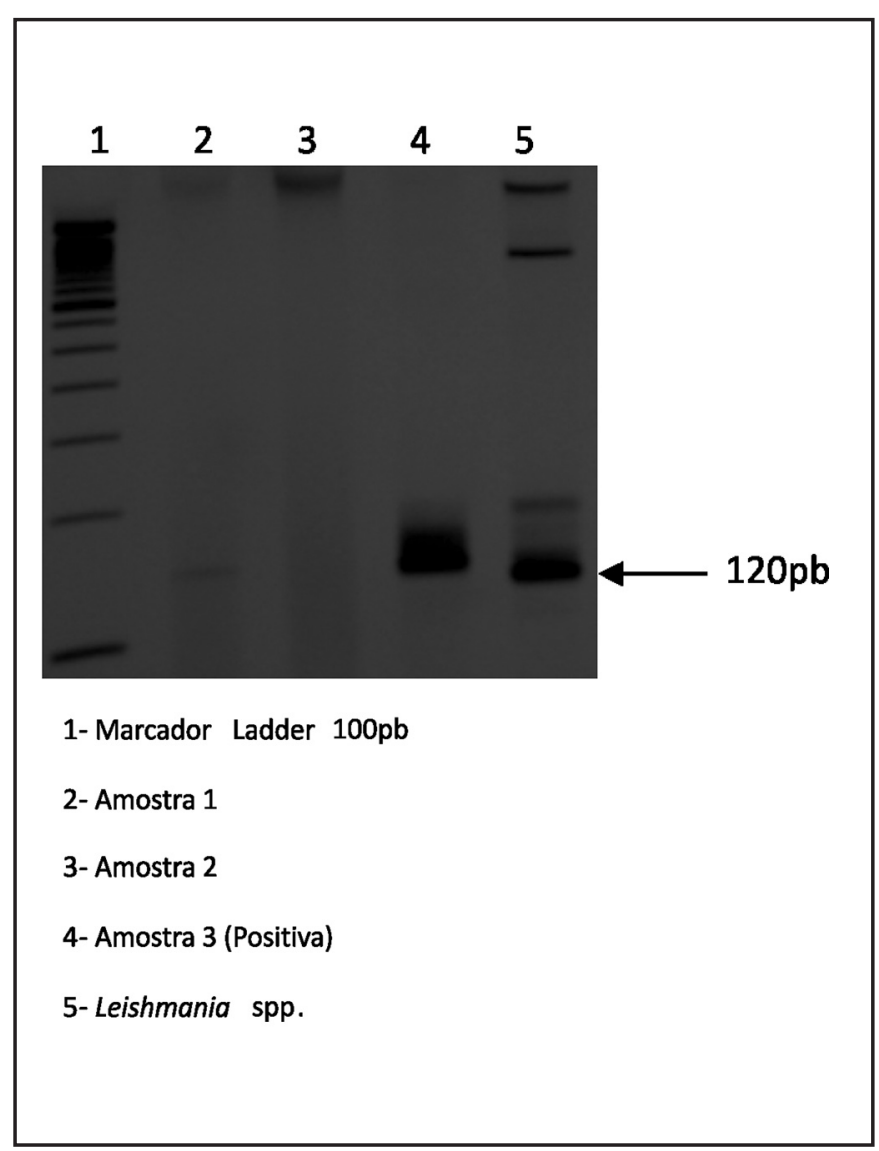

FIGURA 1 - Detecção de Leishmania spp em Lutzomyia cruzi naturalmente infectado.

Para confirmação da espécie de Leishmania, na RFLP, utilizou-se controles positivos de Leishmania (L.) amazonensis, L. (V.) braziliensis e L. (L.) infantum chagasi digeridas com enzima de restrição HaeIII. Constatou-se um padrão de bandas semelhante a $L$. (L.) infantum chagasi na amostra 3 (Figura 2), confirmando a detecção de infecção natural em L. cruzi. 


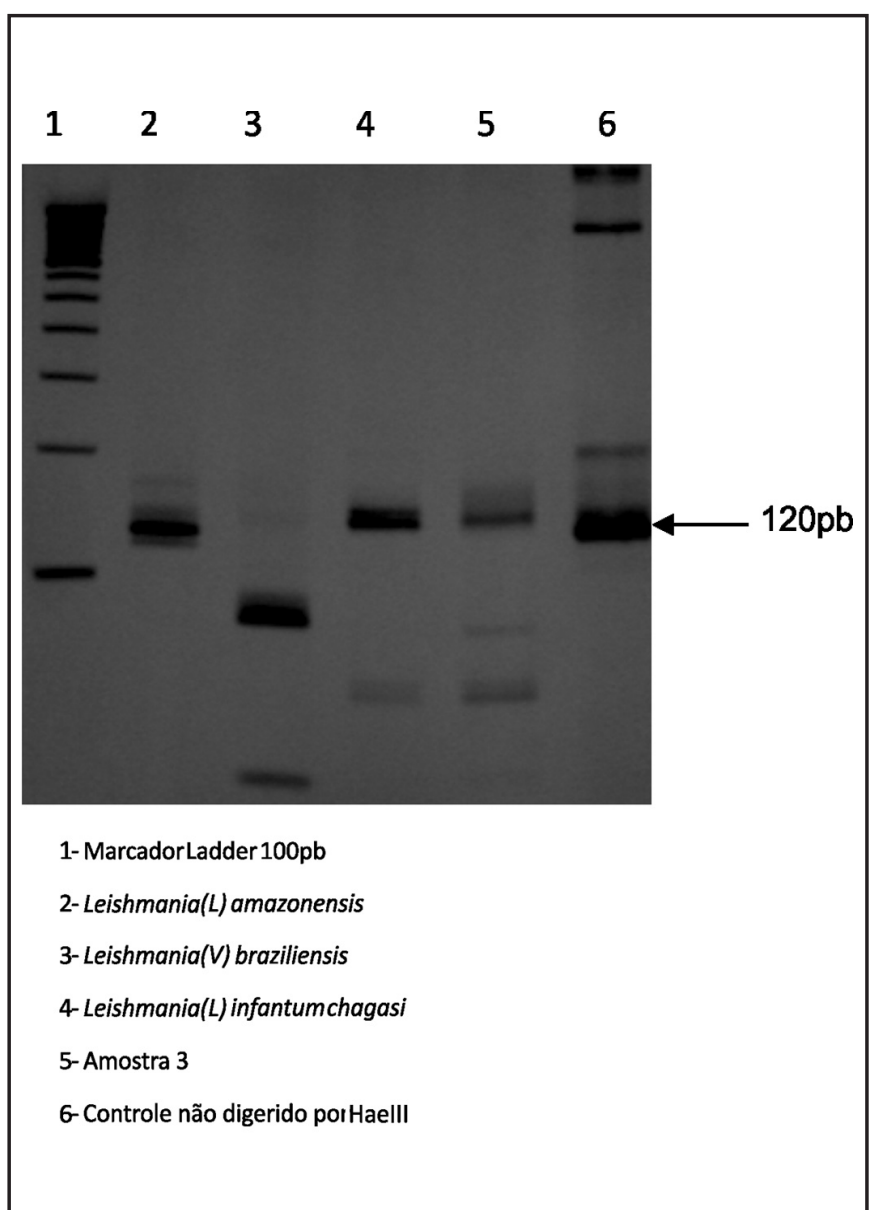

FIGURA 2 - Detecção de Leishmania (L.) infantum chagasi em Lutzomyia cruzi naturalmente infectado.

\section{DIsCUSSÃo}

A distribuição de L. cruzi seria restrita ao Estado de Mato Grosso do $\mathrm{Sul}^{10}$, mas pesquisas entomológicas realizadas no Estado de Mato Grosso, indicam a presença desse vetor nos município de Alto Araguaia, Barão de Melgaço, Barra do Garças, Canarana, Chapada dos Guimarães, Cuiabá, Dom Aquino, Feliz Natal, Itiquira, Jaciara, Juína, Mirassol d'Oeste, Nobres, Nova Brasilândia, Nova Canaã do Norte, Paranatinga, Poconé, Pontal do Araguaia, Poxoréo, Primavera do Leste, Rondonópolis, Rosário Oeste, Santo Antônio do Leverger e Sorriso².

Dentre as espécies de importância médica capturadas, L. cruzi, está incriminada na transmissão de L. (L.) infantum chagasi em Mato Grosso do $\mathrm{Sul}^{4}$ e L. whitmani que está associada à transmissão de L. (V.) braziliensis ${ }^{5}$.

A alta frequência de $L$. cruzi em municípios com alta incidência de casos humanos e taxa de infecção canina de $40 \%$, no período de 1998 a 2005, em Jaciara/MT, sugere possível participação desta espécie na cadeia de transmissão da $\mathrm{LV}^{11}$.

A ocorrência de casos humanos e a presença do L. cruzi, tendo em vista que as fêmeas desta espécie alimentam-se mais facilmente e preferencialmente em humanos ${ }^{12}$, a ocorrência de cães positivos e a ausência de L. longipalpis no município, bem como a detecção de infecção natural por L. (L.) infantum chagasi, evidenciam a participação de L. cruzi na transmissão da LV em Jaciara/MT.

\section{AGRADECIMENTOS}

Agradecemos aos técnicos da Secretaria de Estado de Saúde de Mato Grosso pelos trabalhos entomológicos, imprescindíveis à realização deste trabalho.

\section{CONFLITO DE INTERESSE}

Os autores declaram não haver nenhum tipo de conflito de interesse no desenvolvimento do estudo.

\section{SUPORTE FINANCEIRO}

Secretaria de Estado de Saúde de Mato Grosso (SES/MT), Centro de Pesquisas René Rachou (CPqRR) e Fundação Oswaldo Cruz (FIOCRUZ).

\section{REFERÊNCIAS}

1. Ministério da Saúde. Manual de Vigilância e Controle da Leishmaniose Visceral. Brasília: Fundação Nacional de Saúde; 2003.

2. Missawa NA, Lima GBM. Distribuição espacial de Lutzomyia longipalpis (Lutz \& Neiva, 1912) e Lutzomyia cruzi (Mangabeira, 1938) no Estado de Mato Grosso. Rev Soc Bras Med Trop 2006; 39:337-340.

3. Lainson R, Rangel EF. Ecologia das Leishmanioses. In: Rangel EF, Lainson R, editores. Flebotomíneos do Brasil. Rio de Janeiro: Editora Fiocruz; 2003. p.291-309.

4. Santos SO, Arias J, Ribeiro AA, Hoffmann MP, Freitas RU, Malacco MAF. Incrimination of Lutzomyia cruzi as a vector of American Visceral Leishmaniasis. Med Vet Entomol 1998; 12:315-317.

5. Marcondes CB. Entomologia Médica e Veterinária. São Paulo: Editora Atheneu; 2001.

6. Ferreira JCV. Mato Grosso e seus Municípios. Cuiabá: Editora Buriti; 2001.

7. Michalsky EM, Fortes-Dias CL, Pimenta PFP, Secundino NFC, Dias ES. Avaliação do PCR na investigação de Leishmania sp. em flebotomíneos experimentalmente infectados (Diptera: Psychodidae: Phlebotominae). Rev Inst Med Trop São Paulo 2002; 44:255-259.

8. De Brujin MHL, Barker DC. Diagnosis of New World leishmaniasis specific detection of species of the Leishmania braziliensis complex by amplification of kinetoplast DNA. Acta Tropica 1992; 52:45-58.

9. Pinheiro NA, Moura RP, Monteiro E, Villa LL. Detection of point mutations by non-isotopic single strand conformation polymorphism. Braz J Med Biol Res 1999; 32:55-58.

10. Young D, Duncan MA. Guide to the identification and geografic distribution of Lutzomyia sand files in México, the West Indies, Central and South America (Diptera: Psychodidae). Florida: Associated Publishers American Entomological Institute; 1994.

11. Mestre GLC, Fontes CJF. A expansão da epidemia da leishmaniose visceral no Estado de Mato Grosso, 1998-2005. Rev Soc Bras Med Trop 2007; 40:42-48.

12. Chagas AC, Medeiros JF, Justiniano SCB, Pessoa FAC. Haematophagic behavior in laboratory of Lutzomyia cruzi (Mangabeira) (Diptera: Psychodidae) in relation to three mammalian blood sources in Manaus, Brazil. Acta Amazonica 2007; 37:127-132. 